\title{
Maximum standardized uptake value at pre-treatment PET in estimating lung cancer progression after stereotactic body radiotherapy
}

\author{
Jisun Park, MD¹, Yunseon Choi, MD, PhD², Ki Jung Ahn, MD, PhD², \\ Sung Kwang Park, PhD², Heunglae Cho, MD, PhD², Ji Young Lee, $\mathrm{MD}^{3}$ \\ Departments of ${ }^{1}$ Nuclear Medicine, ${ }^{2}$ Radiation Oncology, Inje University Busan Paik Hospital, \\ Inje University College of Medicine, Busan; ${ }^{3}$ Division of Hematology and Oncology, \\ Department of Internal Medicine, Busan Paik Hospital, Inje University College of Medicine, Busan, Korea
}

\begin{abstract}
Purpose: This study aimed to identify the feasibility of the maximum standardized uptake value (SUVmax) on baseline 18F-fluorodeoxyglucose positron emission tomography-computed tomography (FDG PET/CT) as a predictive factor for prognosis in early stage primary lung cancer treated with stereotactic body radiotherapy (SBRT).

Materials and Methods: Twenty-seven T1-3NOMO primary lung cancer patients treated with curative SBRT between 2010 and 2018 were retrospectively evaluated. Four patients (14.8\%) treated with SBRT to address residual tumor after wedge resection and one patient (3.7\%) with local recurrence after resection were included. The SUVmax at baseline PET/CT was assessed to determine its relationship with prognosis after SBRT. Patients were divided into two groups based on maximum SUVmax on pre-treatment FDG PET/ $\mathrm{CT}$, estimated by receiver operating characteristic curve.

Results: The median follow-up period was 17.7 months (range, 2.3 to 60.0 months). The actuarial 2-year local control, progressionfree survival (PFS), and overall survival were $80.4 \%, 66.0 \%$, and 78.2\%, respectively. With regard to failure patterns, 5 patients exhibited local failure (in-field failure, 18.5\%), 1 (3.7\%) experienced regional nodal relapse, and other 2 (7.4\%) developed distant failure. SUVmax was significantly correlated with progression ( $p=0.08$, optimal cut-off point SUVmax $>5.1)$. PFS was significantly influenced by pretreatment SUVmax (SUVmax $>5.1$ vs. SUVmax $\leq 5.1 ; p=0.012$ ) and T stage (T1 vs. T2-3; $p=0.012$ ).

Conclusion: SUVmax at pre-treatment FDG PET/CT demonstrated a predictive value for PFS after SBRT for lung cancer.
\end{abstract}

Keywords: Lung neoplasms, Positron-emission tomography, Standardized uptake value, Stereotactic body radiotherapy, Progressionfree survival

\section{Introduction}

Stereotactic body radiotherapy (SBRT) is used for curative treatment when surgery is not possible in patients with early stage lung cancer [1]. SBRT may be considered a first-line treatment modality for elderly patients with other serious diseases, such as restrictive/obstructive lung disease or double primary cancer, or for patients who are postoperative resection

Received 03 January 2019, Revised 15 February 2019, Accepted 13 March 2019.

Correspondence: Yunseon Choi, MD, PhD, Department of Radiation Oncology, Inje University Busan Paik Hospital, Inje University College of Medicine, 75 Bokji-ro, Busanjin-gu, Busan 47392, Korea. Tel: +82-51-890-8606, Fax: +82-51-891-1754, E-mail: rtyoon@gmail.com (http://orcid.org/0000-0002-1397-6753)

(c) This is an Open Access article distributed under the terms of the Creative Commons Attribution Non-Commercial License (http://creativecommons.org/ licenses/by-nc/4.0/) which permits unrestricted non-commercial use, distribution, and reproduction in any medium, provided the original work is properly cited.

www.e-roj.org 
margin-positive or exhibit suspicious local recurrence [2]. SBRT, a hypo-fractionated high-dose radiotherapy (RT) introduced by Timmerman et al. [1] in the late 2000s, has dramatically altered contemporary methods of RT.

SBRT has demonstrated its ability to control tumors more effectively than conventional fractionated RT in cases with lower tumor burden. In addition to direct cell killing by radiation, SBRT also kills tumor cells in indirect ways, such as collapse of the tumor vascular structure and altering the tumor microenvironment [3]. In primary lung cancer, the outcome of SBRT is fairly good, affording the same level of local control as surgery. However, the proportion of distant metastasis is higher than that of surgery [4]. Thus, SBRT has not been able to completely replace lobectomy, despite the advantages in quality of life in patients with early stage nonsmall cell lung cancer.

In the era of SBRT, the role of $18 \mathrm{~F}$-fluorodeoxyglucose positron emission tomography-computed tomography (FDG PET/CT) has emerged. In terms of detecting cancer, FDG PET/ $\mathrm{CT}$ can be used to confirm the therapeutic response of SBRT or to confirm relapse $[5,6]$. Additionally, Clarke et al. [7] reported that the maximum standardized uptake value (SUVmax) in primary FDG PET/CT can be used to assess prognosis. These results demonstrated that progression-free survival (PFS) and distant metastasis-free survival could be affected by the metabolic activity of the tumor before SBRT [7].

In this study, we aimed to analyze the relationship between the SUVmax of baseline FDG PET/CT and treatment outcome after SBRT. There has been controversy whether the SUV of pretreatment FDG PET/CT determines prognosis [8]. Although there are reports that SUVmax is not helpful in predicting prognosis, recent meta-analyses $[9,10]$ have recognized and bolstered the predictive value of SUVmax in FDG PET/CT. The fact that higher SUVmax is related to a less favorable prognosis has already been reported by Lee et al. [11], SUVmax 5 (the cutoff value used in the current study) is a baseline value reported in previous studies that appeared to determine the prognosis for distant metastasis [7] in SBRT.

In this study, we especially focused on the correlation between PFS and SUVmax values. In addition, we evaluated clinical factors, such as T stage or tumor size [12-15], which we expected to be related to prognosis. Local control (LC) and distant metastasis after treatment were also evaluated.

\section{Materials and Methods}

Twenty-seven patients who underwent baseline FDG PET/CT,
Table 1. Patients' characteristics

\begin{tabular}{lc}
\hline \multicolumn{1}{c}{ Characteristic } & Value \\
\hline Age (yr) & $73.0(60.0-84.0)$ \\
Gender & \\
Male & $21(77.8)$ \\
Female & $6(22.2)$ \\
T stage & \\
T1 & $19(70.4)$ \\
T2 & $5(18.5)$ \\
T3 & $3(11.1)$ \\
Tumor size (cm) & $2.0(1.0-6.7)$ \\
Histology & \\
Adenocarcinoma & $140(51.9)$ \\
Squamous cell carcinoma & $12(44.4)$ \\
Not obtained & $1(3.7)$ \\
Previous resection & \\
Yes & $5(18.5)$ \\
No & $22(81.5)$ \\
SUVmax & \\
$\leq 5.1$ & $11(40.7)$ \\
$>5.1$ & $16(59.3)$ \\
\hline
\end{tabular}

Values are presented as median (range) or number (\%). SUVmax, maximum standardized uptake value.

among those who underwent SBRT treatment for primary lung cancer (CT1-3NOM0) at at Inje University Busan Paik Hospital, between January 2010 and May 2018, were analyzed. The Institutional Review Board of Busan Paik Hospital approved this study (No. 18-0199). Informed consent has been waived by the IRB because it was a retrospective chart review study. All patients underwent FDG PET/CT before SBRT to diagnose lung lesions. Additionally, all patients, except for one, underwent pathological diagnosis for the lung lesion before undergoing SBRT. TNM stage was designated according to the American Joint Committee on Cancer Staging 8th edition. N staging was based on pre-treatment PET/CT results. Mediastinal lymph node evaluation using endobronchial ultrasound guided transbronchial needle aspiration (EBUS-TBNA) or mediastinoscopy was not essentially conducted for this study. Tumors were divided into central and peripheral tumors according to the RTOG 0236 protocol [1]: central tumor was defined as a tumor located within $2 \mathrm{~cm}$ from the secondary branch of the proximal bronchus. Tumor response after SBRT was assessed by Response Evaluation Criteria in Solid Tumors (RECIST) criteria version 1.1.

For SBRT, all patients were treated using the Varian RapidArc 
A

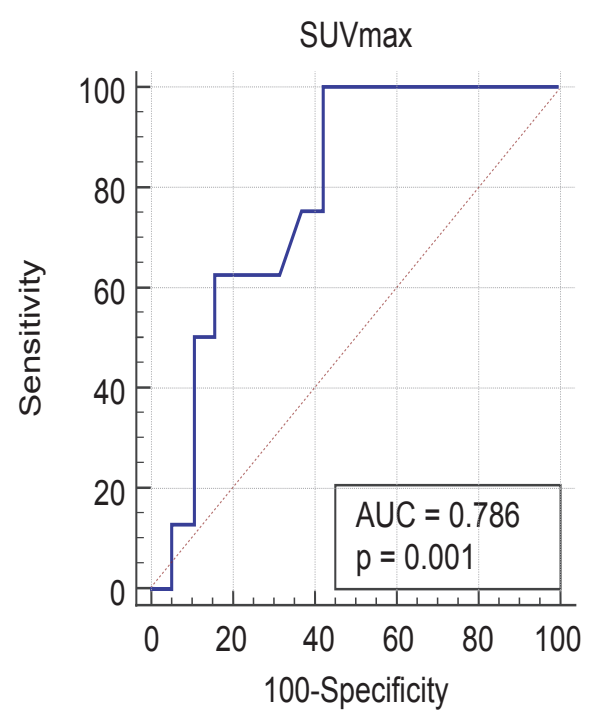

B

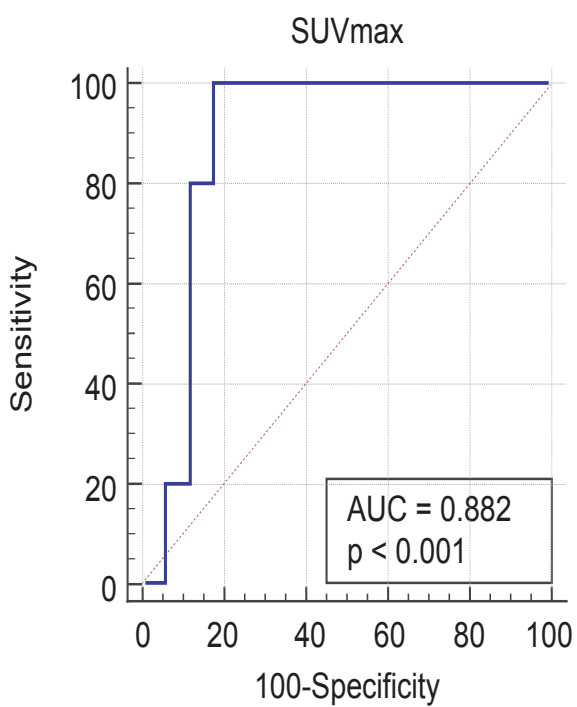

Fig. 1. Cutoff point for SUVmax in receiver operating characteristic curve for all patients (A) and for previously unresected patients (B). AUC, area under curve.

system (Varian Medical Systems Inc., Palo Alto, CA, USA). In the treatment plan, four-dimensional CT was performed to reflect movement by breathing. SBRT was performed in doses of $\geq 12$ Gy per fraction. The total doses of $45 \mathrm{~Gy}, 48 \mathrm{~Gy}, 54 \mathrm{~Gy}$, and $60 \mathrm{~Gy}$ were administered, divided into 3-5 fractions. The median total dose was $54 \mathrm{~Gy}$. Biologically effective doses of all patients were $>100 \mathrm{~Gy}_{10}$, which is known as the dose level for higher LC [16]. Large tumors (T2-3 stage) were generally irradiated with a small fraction size to reduce the pulmonary toxicity. Conversely, in the cases with tumor resection, the total radiation dose and fraction number were reduced. Most patients underwent first-line SBRT with the aim of curative local treatment. However, one patient (3.7\%) was treated using SBRT for local recurrence after resection; other four (14.8\%), who were treated with SBRT to address residual tumor after wedge resection, were also included.

After 6 hours of fasting, patients were injected with 4.44 $\mathrm{MBg} / \mathrm{kg}$ of F-18 FDG for PET/CT scan. One hour after injection, patients underwent FDG PET/CT (Discovery STE; GE Healthcare, Milwaukee, WI, USA). In addition, the SUV was obtained and quantified using the AW VolumeShare 5 Workstation (GE Healthcare). FDG PET/CT parameters were collected and used for analysis. Patients were divided into two groups using a calculated cutoff SUVmax value.

Follow-up (FU) evaluation was performed by imaging test at 1 month, 3 months, and 6 months after SBRT, and additional outpatient clinic FU was performed more than once every 6 months thereafter. Local failure (progression) was defined as a least $20 \%$ of increase of diameter of target lesion. LC was measured in terms of time from the start of SBRT to local failure or last FU. PFS was measured on the basis of time from the first SBRT start date to any progression or last FU. Overall survival (OS) was defined as time from SBRT start to death or time to last FU. PFS was analyzed as a primary endpoint. In addition, the overall progression rate, $\mathrm{LC}$, and distant metastasis rate according to the FDG PET/CT results were also analyzed.

Statistical analyses were performed using SPSS version 20.0 (IBM Corporation, Armonk, NY, USA). The chi-squared test was initially used to determine prognostic factor(s) associated with progression. The Youden index was used to estimate the cutoff point in the receiver operating characteristic (ROC) curve (MedCalc software version 18.9; MedCalc, Mariakerke, Belgium). Kaplan-Meier analysis and log-rank test were used for survival analysis. Cox regression was used to determine an independent prognostic factor. A two-tailed $p<0.05$ was considered to be statistically significant.

\section{Results}

\section{Patients and treatment}

Patient characteristics are summarized in Table 1. The median age of the patients was 73.0 years (range, 60 to 84 years). All patients were $>60$ years of age, and males comprised 
Table 2. Lung cancer progression according to clinical factors

\begin{tabular}{|c|c|c|c|}
\hline & \multirow{2}{*}{$\begin{array}{c}\text { Controlled } \\
\text { No. }\end{array}$} & \multicolumn{2}{|c|}{ Progressed } \\
\hline & & No. & $p$-value \\
\hline Age (yr) & & & 0.678 \\
\hline$\leq 73$ & 9 & 5 & \\
\hline$>73$ & 10 & 3 & \\
\hline Gender & & & 0.633 \\
\hline Male & 13 & 7 & \\
\hline Female & 6 & 1 & \\
\hline T stage & & & 0.027 \\
\hline $\mathrm{T} 1$ & 16 & 3 & \\
\hline T2-3 & 3 & 5 & \\
\hline Tumor size (cm) & & & 0.420 \\
\hline$\leq 2.0$ & 11 & 3 & \\
\hline$>2.0$ & 8 & 5 & \\
\hline Previous resection & & & 0.136 \\
\hline Yes & 2 & 3 & \\
\hline No & 17 & 5 & \\
\hline SUVmax & & & 0.008 \\
\hline$\leq 5.1$ & 11 & 0 & \\
\hline$>5.1$ & 8 & 8 & \\
\hline
\end{tabular}

SUVmax, maximum standardized uptake value.

Table 3. Univariate analysis for actuarial PFS

\begin{tabular}{|c|c|c|c|}
\hline & 1-yr PFS (\%) & 2-yr PFS (\%) & $p$-value \\
\hline Age (yr) & & & 0.894 \\
\hline$\leq 73$ & 81.5 & 58.2 & \\
\hline$>73$ & 90.0 & 77.1 & \\
\hline Gender & & & 0.067 \\
\hline Male & 81.0 & 56.1 & \\
\hline Female & 100 & 100 & \\
\hline T stage & & & 0.012 \\
\hline $\mathrm{T} 1$ & 100 & 88.9 & \\
\hline T2-3 & 51.4 & 17.1 & \\
\hline Tumor size (cm) & & & 0.292 \\
\hline$\leq 2.0$ & 100 & 87.5 & \\
\hline$>2.0$ & 70.7 & 47.1 & \\
\hline Previous resection & & & 0.632 \\
\hline Yes & 82.1 & 54.7 & \\
\hline No & 100 & 100 & \\
\hline SUVmax & & & 0.012 \\
\hline$\leq 5.1$ & 100 & 100 & \\
\hline$>5.1$ & 77.8 & 46.7 & \\
\hline
\end{tabular}

PFS, progression-free survival; SUVmax, maximum standardized uptake value.
$77.8 \%(n=21)$ of the cohort. Slightly more than one-third of the patients $(n=11)$ did not undergo radical surgery before SBRT because of existing double primary cancer. With regard to other comorbidities, two patients had heart failure, two had obstructive lung disease, and one had a restrictive lung disease. Tumor was located in the right lobe in 20 patients (74.0\%) and in the left lobe in 7 (25.9\%). There were 20 $(67.7 \%)$ cases of tumor located in the upper or middle lobe and $10(33.3 \%)$ cases in the lower lobe. In this study, the all lung tumors belonged to the peripheral lesion ( $>2 \mathrm{~cm}$ from tracheo-proximal bronchial tree, 100\%) based on RTOG 0236 protocol [1]. Staging was T1 in 19, T2 in 5, and T3 in 3. No patients had lymph node or distant metastasis at the start of SBRT. The median tumor size was $2.0 \mathrm{~cm}$ (range, 1 to 6.7 $\mathrm{cm})$. In the baseline FDG PET/CT, the median SUVmax was 5.7 (range, 1.8 to 21.4). Depending on the pathological subtype, there were 14 cases of adenocarcinoma, 12 cases of squamous cell carcinoma, and one case in which a tissue sample was not obtained.

\section{Patterns of failure}

The median FU period of the patients was 17.7 months (range, 2.3 to 60.0 months). In addition, the median FU period for surviving patients was 15.9 months. The response rate was $33.3 \%(n=9)$ and all responders exhibited partial response. Overall, 8 patients (29.6\%) exhibited progression during the FU period. According to failure patterns, local failure occurred in 5 patients (18.5\%) while regional lymph nodal failure occurred in 1 (3.7\%). Distant metastases were observed in 2 (7.4\%) cases. With regard to the metastatic sites, one patient exhibited brain metastasis and the other exhibited contralateral lung metastasis.

During the FU period, 7 patients (25.9\%) died: 3 patients of lung cancer progression; 2 of heart failure; and the remaining 2 of intercurrent disease. Regarding treatment toxicity, 3 patients $(11.1 \%)$ received oral steroids for radiation pneumonitis after treatment; however, all eventually recovered.

\section{Factors related to progression}

Using progression and SUVmax data, the optimal cutoff value was obtained using ROC curve analysis. SUVmax $>5.1$ in baseline FDG PET/CT (area under the ROC curve $[A U C]=0.786$, $p=0.001$, sensitivity $100 \%$, specificity $57.9 \%$ ) was calculated as the optimal cutoff point to predict patient progression (Fig. $1 \mathrm{~A})$. In the further subgroup analysis for patients without previous resection history $(n=22)$, the optimal cutoff point for progression was calculated as SUVmax $>7.6$ (AUC $=0.882$, 
A

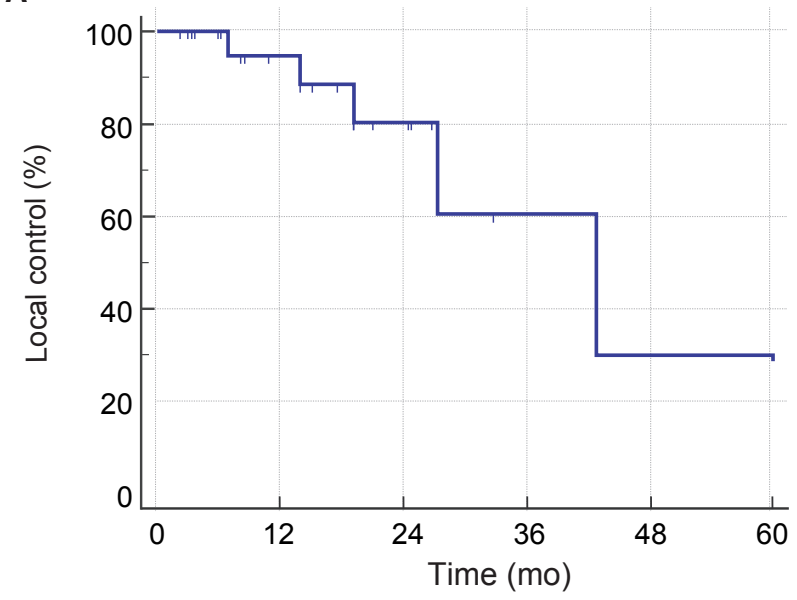

B

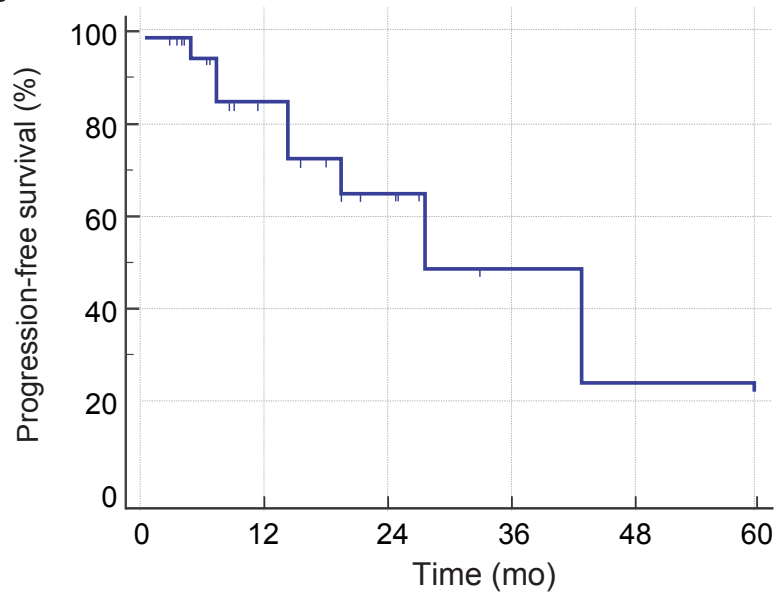

C

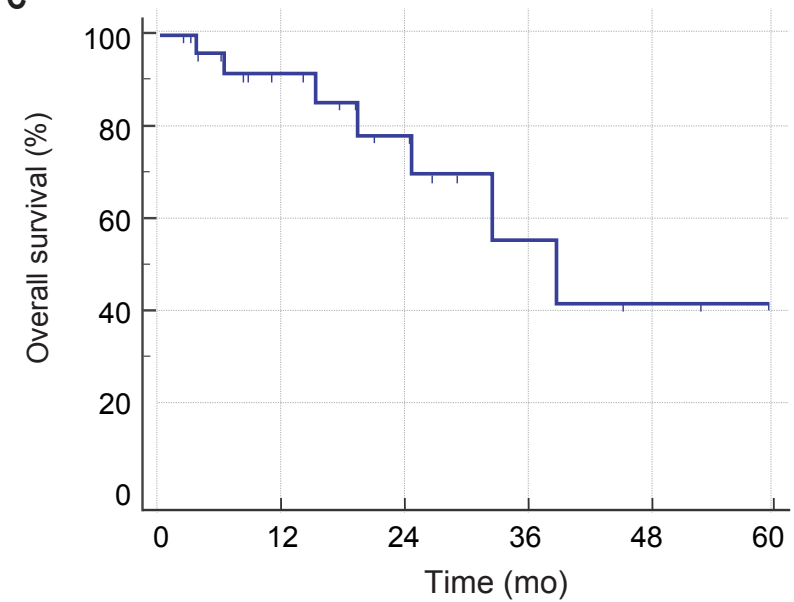

Fig. 2. Two-year local control (A), progression-free survival (B), and overall survival (C).

$p<0.001$, sensitivity $100 \%$, specificity $82.4 \%$ ) (Fig. 1B).

The occurrence of progression according to clinical factors are summarized in Table 2. SUVmax was significantly correlated with progression $(p=0.008)$. Patients with advanced T stage (i.e., T2-3) also exhibited higher overall progression $(p=0.027)$.

\section{Prognostic factors for PFS}

The actuarial 1-year LC, PFS, and OS were $94.7 \%, 85.6 \%$, and 91.4\%, respectively. Two-year LC was $80.4 \%$ and 2-year PFS was $66.0 \%$ (Fig. 2A, 2B). The 2-year OS was $78.2 \%$ (Fig. 2C).

Differences in PFS according to clinical factors are shown in Table 3. In univariate analysis, PFS was significantly associated with T stage and SUVmax at baseline FDG PET/CT. PFS was significantly influenced by T stage (T1 vs. T2-3; $p=0.012$ ) (Table 3 ) and pre-treatment SUVmax (SUVmax $>5.1$ vs. SUVmax $\leq 5.1$ $p=0.012$ ) (Fig. 3). Moreover, OS was not significantly affected by T stage $(p=0.993)$ or pre-treatment SUVmax $(p=0.716)$.

\section{Discussion and Conclusion}

The most important finding of this study was that pretreatment SUVmax was an important factor in determining prognosis of lung cancer. This study showed the influence of cellular metabolism on prognosis of lung cancer after SBRT. We suggested adequate cutoff points of the SUVmax value in predicting the prognosis of lung cancer. More specifically, a cutoff SUVmax value of 5.1 demonstrated a role in determining PFS. High FDG uptake of tumor is a reflection of aggressiveness. Patients with SUVmax $>5.1$ at baseline FDG PET/CT exhibited continuously decreasing in PFS over time. This indicates that there is a need for long-term close FU with the possibility of recurrence in these patients with higher SUVmax. On the other hand, compared with previous studies investigating SBRT $[14,17,18]$, our study demonstrated that

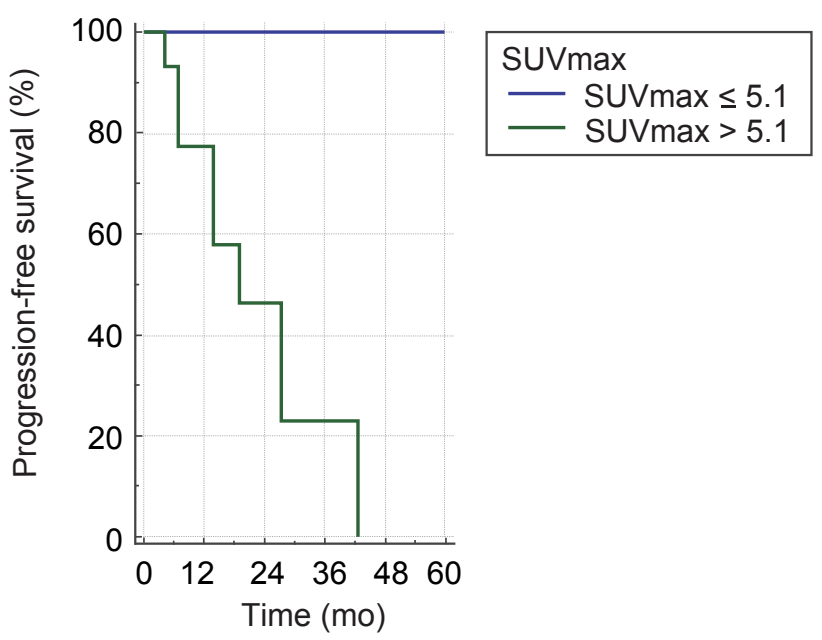

Fig. 3. Progression-free survival by SUVmax of baseline positron emission tomography-computed tomography $(p=0.012)$. 
Table 4. Summary of patients with treatment failures

\begin{tabular}{ccccccc}
\hline $\begin{array}{c}\text { Gender/age } \\
(\mathrm{yr})\end{array}$ & $\begin{array}{c}\text { Tumor size } \\
(\mathrm{cm})\end{array}$ & SUVmax & $\begin{array}{c}\text { Resection } \\
\text { before RT }\end{array}$ & $\begin{array}{c}\text { RT dose } \\
(\mathrm{Gy} / \mathrm{fx})\end{array}$ & Failure pattern & $\begin{array}{c}\text { Progression- } \\
\text { free interval (mo) }\end{array}$ \\
\hline M/81 & 1.5 & 5.6 & Yes (recur) & $60 / 3$ & LF & 13.9 \\
M/67 & 2.4 & 14.2 & No & $60 / 4$ & LF & 19.2 \\
M/80 & 6.7 & 8.6 & No & $60 / 5$ & LF & 7.0 \\
F/74 & 1.1 & 6.5 & Yes (R2) & $45 / 3$ & LF & 27.3 \\
M/69 & 1.4 & 5.3 & Yes (R2) & $45 / 3$ & LF & 42.7 \\
M/73 & 3.9 & 16.9 & No & $60 / 3$ & RF (lymph node) & 7.0 \\
M/70 & 3 & 11.2 & No & $54 / 3$ & DF (brain) & 14.0 \\
M/66 & 4.6 & 9.7 & No & $48 / 4$ & DF (contralateral lung) & 26.8 \\
\hline
\end{tabular}

SUVmax, maximum standardized uptake value; RT, radiotherapy; LF, local failure; RF, regional failure; DF, distant failure.

distant metastasis was not a dominant failure pattern. The lower distant metastasis rate of this study may be related with the short median follow-up period (17.7 months). Inversely, this study showed a higher local failure rate compared to the previous study by Baumann et al. [14]. To overcome the risk of local failure, additional local treatment could be considered (such as post-radiation resection or radiofrequency ablation [19]) in patients with large primary tumor size or higher SUVmax. Table 4 shows the detailed information about the progressed patients. The total radiation dose for half of resected lung cancer patients was relatively lower than other patients (Table 4). It should be noted that there were more recurrences in those patients treated with $45 \mathrm{~Gy} / 3 \mathrm{fx}$ SBRT after incomplete wedge resection (Table 4). According to this study, we should use full dose of radiation for postoperative SBRT.

Plus, the patient who underwent SBRT for local recurrence eventually relapsed again. In addition, one third of T3 patients ( $n=1$, tumor size $=6.7 \mathrm{~cm}$ ) experienced local failure.

Based on the fact that pretreatment FDG PET/CT was helpful in determining the possibility of progression, it is reasonable to believe that serial FDG PET/CT (before and after SBRT) may be helpful in predicting patient outcome after treatment. Several studies have already reported that FDG PET/CT after SBRT was helpful in predicting prognosis $[5,11,20,21]$. In particular, FDG $\mathrm{PET} / \mathrm{CT}$ can be useful to confirm local recurrence [6]. In this study, CT was performed mainly during patient FU because of the economic burden to the patient and the national health insurance policy of Korea. However, it is clear that posttreatment FDG PET/CT can be a good alternative diagnostic tool. If FDG PET/CT had a more reasonable cost, it could be applied during patient FU instead of CT. Conversely, the SUVmax of FDG PET/CT was not an independent prognostic factor according to the results of this study; thus, there is a limit to its diagnostic value. Therefore, efforts to develop a more sensitive diagnostic tool that can complement FDG PET/ CT should be pursued.

Regarding limitations of this study, the number of patients in our institution was relatively small, which may have introduced selection bias. This study has limited statistical power because of small number of patients. Patients of SUVmax $<5.1$ showed no progressions in this study. However, it may reflect the bias from the small sample size. A multiinstitutional study, therefore, could overcome this drawback. Additionally, because it was a retrospective study, the patient group was not homogeneous. Patients in the terminal stage are often referred to a nursing home or other hospital. For this reason, the accuracy of OS estimates tended to be low. Therefore, although this study demonstrated a fairly good OS, the results require careful, if not cautious, interpretation and application.

In conclusion, SUVmax at pre-treatment FDG PET/CT had a predictive value for PFS. FDG PET/CT can be a useful and credible modality for estimating prognosis in patients with primary lung cancer after SBRT.

\section{Conflict of Interest}

No potential conflict of interest relevant to this article was reported.

\section{References}

1. Timmerman R, McGarry R, Yiannoutsos $C$, et al. Excessive toxicity when treating central tumors in a phase II study of stereotactic body radiation therapy for medically inoperable 
early-stage lung cancer. J Clin Oncol 2006;24:4833-9.

2. Videtic GMM, Donington J, Giuliani M, et al. Stereotactic body radiation therapy for early-stage non-small cell lung cancer: Executive Summary of an ASTRO Evidence-Based Guideline. Pract Radiat Oncol 2017;7:295-301.

3. Kim MS, Kim W, Park IH, et al. Radiobiological mechanisms of stereotactic body radiation therapy and stereotactic radiation surgery. Radiat Oncol J 2015;33:265-75.

4. Chi A, Liao Z, Nguyen NP, Xu J, Stea B, Komaki R. Systemic review of the patterns of failure following stereotactic body radiation therapy in early-stage non-small-cell lung cancer: clinical implications. Radiother Oncol 2010;94:1-11.

5. Pastis NJ Jr, Greer TJ, Tanner NT, et al. Assessing the usefulness of 18F-fluorodeoxyglucose PET-CT scan after stereotactic body radiotherapy for early-stage non-small cell lung cancer. Chest 2014;146:406-11.

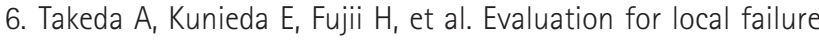
by $18 \mathrm{~F}-\mathrm{FDG} \mathrm{PET} / \mathrm{CT}$ in comparison with $\mathrm{CT}$ findings after stereotactic body radiotherapy (SBRT) for localized non-smallcell lung cancer. Lung Cancer 2013;79:248-53.

7. Clarke K, Taremi M, Dahele M, et al. Stereotactic body radiotherapy (SBRT) for non-small cell lung cancer (NSCLC): is FDG-PET a predictor of outcome? Radiother Oncol 2012;104:62-6.

8. Burdick MJ, Stephans KL, Reddy CA, Djemil T, Srinivas SM, Videtic GM. Maximum standardized uptake value from staging FDG-PET/CT does not predict treatment outcome for earlystage non-small-cell lung cancer treated with stereotactic body radiotherapy. Int J Radiat Oncol Biol Phys 2010;78:10339.

9. Na F, Wang J, Li C, Deng L, Xue J, Lu Y. Primary tumor standardized uptake value measured on F18Fluorodeoxyglucose positron emission tomography is of prediction value for survival and local control in non-smallcell lung cancer receiving radiotherapy: meta-analysis. J Thorac Oncol 2014;9:834-42.

10. Dong M, Liu J, Sun $X$, Xing L. Prognositc significance of SUV(max) on pretreatment (18) F-FDG PET/CT in early-stage non-small cell lung cancer treated with stereotactic body radiotherapy: a meta-analysis. J Med Imaging Radiat Oncol 2017;61:652-9.

11. Lee DS, Kim YS, Yoo IR, et al. Long-term clinical experience of high-dose ablative lung radiotherapy: high pre-treatment
[18F]fluorodeoxyglucose-positron emission tomography maximal standardized uptake value of the primary tumor adversely affects treatment outcome. Lung Cancer 2013;80:172-8.

12. Lee $S$, Song SY, Kim SS, et al. Feasible optimization of stereotactic ablative radiotherapy dose by tumor size for stage I non-small-cell lung cancer. Clin Lung Cancer 2018;19:e253-e261.

13. Park S, Urm S, Cho H. Analysis of biologically equivalent dose of stereotactic body radiotherapy for primary and metastatic lung tumors. Cancer Res Treat 2014;46:403-10.

14. Baumann P, Nyman J, Hoyer $M$, et al. Outcome in a prospective phase II trial of medically inoperable stage I nonsmall-cell lung cancer patients treated with stereotactic body radiotherapy. J Clin Oncol 2009;27:3290-6.

15. Fakiris AJ, McGarry RC, Yiannoutsos CT, et al. Stereotactic body radiation therapy for early-stage non-small-cell lung carcinoma: four-year results of a prospective phase II study. Int J Radiat Oncol Biol Phys 2009;75:677-82.

16. Onishi H, Araki T, Shirato $H$, et al. Stereotactic hypofractionated high-dose irradiation for stage I nonsmall cell lung carcinoma: clinical outcomes in 245 subjects in a Japanese multiinstitutional study. Cancer 2004;101:1623-31.

17. Senthi S, Lagerwaard FJ, Haasbeek CJ, Slotman BJ, Senan S. Patterns of disease recurrence after stereotactic ablative radiotherapy for early stage non-small-cell lung cancer: a retrospective analysis. Lancet Oncol 2012;13:802-9.

18. Bradley JD, El Naqa I, Drzymala RE, Trovo M, Jones G, Denning MD. Stereotactic body radiation therapy for early-stage nonsmall-cell lung cancer: the pattern of failure is distant. Int J Radiat Oncol Biol Phy. 2010;77:1146-50.

19. Palussiere J, Chomy F, Savina M, et al. Radiofrequency ablation of stage IA non-small cell lung cancer in patients ineligible for surgery: results of a prospective multicenter phase II trial. J Cardiothorac Surg 2018;13:91.

20. Bollineni VR, Widder J, Pruim J, Langendijk JA, Wiegman EM. Residual 18F-FDG-PET uptake 12 weeks after stereotactic ablative radiotherapy for stage I non-small-cell lung cancer predicts local control. Int J Radiat Oncol Biol Phys 2012;83:e551-5.

21. Hoopes DJ, Tann M, Fletcher JW, et al. FDG-PET and stereotactic body radiotherapy (SBRT) for stage I non-smallcell lung cancer. Lung Cancer 2007;56:229-34. 